\title{
AEROFOTOGRAMETRIA DE PEQUENO FORMATO APLICADA A REALIZAÇÃO DA CARTOGRAFIA BÁSICA DA CIDADE DE PALMEIRINA-PE
}

\author{
Fernando da Silva Alexandre ${ }^{(a)}$, Renilson Pinto da Silva Ramos ${ }^{(b)}$, Rodolfo Alexandre da \\ Silva Gomes de Deus ${ }^{(\mathrm{c})}$, Daniel Dantas Moreira Gomes ${ }^{(\mathrm{d})}$
}

\begin{abstract}
(a) Geografia/ Universidade de Pernambuco, Campus Garanhuns, fnando257@gmail.com
(b)Geografia/ Universidade de Pernambuco, Campus Garanhuns, renilsonr5@ hotmail.com

${ }^{(c)}$ Geografia/ Universidade de Pernambuco, Campus Garanhuns, rdolfodeus@ gmail.com

${ }^{(d)}$ Geografia/ Universidade de Pernambuco, Campus Garanhuns, daniel.gomes@upe.br
\end{abstract}

\section{Eixo: GEOTECNOLOGIAS E MODELAGEM ESPACIAL EM GEOGRAFIA FÍSICA}

\begin{abstract}
RESUMO
Com a enorme demanda de informações espaciais georreferenciadas para o planejamento e administração urbana, é necessário que se tenha no mínimo a planta cadastral com seus arruamentos, construções imobiliárias urbanas e outros elementos que compõem o espaço físico para facilitar o gerenciamento, principalmente nas cidades de pequeno porte, que há a ausência de um plano diretor. Neste trabalho foi utilizado o VANT Phantom 3 Advanced, equipado com uma câmera Phantom Vision de 12 Megapixels, com a junção de um SIG, foi possivel realizer o mapeamento da cidade de Palmeirina-PE, utilizou-se uma metodologia que propriciasse obter os resultados desejados e que se adequassem à realidade do objeto de estudo.
\end{abstract}

Palavras chave: aerofotogrametria, cadastro multifinalitário, Palmeirina-PE

\section{Introdução}

Com a enorme demanda de informações espaciais georreferenciadas para o planejamento e administração urbana, é necessário que se tenha no mínimo a planta cadastral com seus arruamentos, construções imobiliárias urbanas e outros elementos que compõem o espaço físico para facilitar o gerenciamento, principalmente nas cidades de pequeno porte, que há a ausência de um plano diretor.

Mas para obter tais dados é indispensável uma base cartográfica sólida, que dependendo de sua qualidade poderá comprometer todo o mapeamento da mancha urbana e a realização do cadastro urbano multifinalitário (CUM), segundo Souza (2001) o cadastro urbano multifinalitário é composto por uma parte cartográfica, que deverá compor uma planta básica da área em questão, e uma parte descritiva com informações sobre elementos diversos como imóveis e seus proprietários, infraestrutura entre outros.

O Cadastro urbano multifinalitário (CUM) é um sistema de informações, de caráter local, que se destina a subsidiar o planejamento, a tributação, o licenciamento de atividades, a fiscalização e 
OS DESAFIOS DA GEOGRAFIA FÍSICA NA FRONTEIRA DO CONHECIMENTO

Instituto de Geociências - Unicamp

Campinas - SP

28 de Junho à 02 de Julho de 2017

todas as funções que emanam das competências dos municípios relacionadas à gestão espaço urbano, carecendo de constantes atualizações.

Para a realização do CUM é necessário ter o levantamento em campo da área a ser mapeada, e um dos melhores meios de se fazer tal levantamento é com o auxílio do sensoriamento remoto, que é a melhor técnica para a obtenção de dados de grandes áreas e com menor gasto monetário (ROSA, 2009), sabendo-se que para a realização do Cadastro Urbano Multifinalitário é de grande importância a qualidade das cenas trabalhadas, portanto o meio mais viável de se obter essas cenas é a partir da Aerofotogrametria.

Atualmente o melhor meio para a realização da aerofotogrametria em pequenas áreas como cidades, são os Veículos Aéreos não Tripulados (VANTs), que por seu baixo custo operacional e trabalhar de forma automática, não de forma autônoma, propiciará a obtenção de cenas com grande qualidade na resolução temporal e espacial, o que irá influenciar diretamente na fotointerpretação e nos resultados. Agregando toda as informações obtidas em campo a um Sistema de Informações Geográfica (SIG), que permitirá fazer um estudo mais minucioso da localidade, viabilizando o gerenciamento, criação, armazenamento e manuseio desses dados.

Este trabalho tem como principal objetivo realizar somente a cartografia básica da mancha urbana do município de Palmeirina-PE, figura 1, para a posterior consolidação do CUM, utilizando a aerofotogrametria em conjunto com o Geoprocessamento, para posteriormente avaliar a viabilidade na utilização dos VANTs para a realização do CUM.

\section{Metodologias}

\subsection{Materiais}

Inicialmente fez-se o levantamento bibliográfico e material cartográfico base. Foram consultados, livros, artigos, teses, monografias, dissertações e trabalhos técnicos, para dar fomento teórico a pesquisa.

Neste trabalho utilizou-se o VANT da fabricante chinesa DJI, Phantom 3 Advanced, equipado com uma camera Phantom Vision de 12 Megapixels, equipado com o sistema GPS e Glonass, com tempo de vôo de aproximadamente 23 minutos por bateria, sua escolha deu-se por ser um veiculo autonomatico de baixo custo. O mesmo possibilitou a obtenção de 784 cenas em 11 voos, a uma altitude média de 100 metros a cima do nivel do solo, seguindo a orientação legal vigente, que inplica um voo a uma altura máxima de 120 metros a cima do nível do solo (SILVA et al., 2015). 
XVII Simpósio Brasileiro

de Geografia Fisica Aplicada

I Congresso Nacional

de Geografia Física

\section{OS DESAFIOS DA GEOGRAFIA FÍSICA NA FRONTEIRA DO CONHECIMENTO Instituto de Geociências - Unicamp \\ Campinas - SP \\ 28 de Junho à 02 de Julho de 2017}

Os dados cartográficos foram armazenados no ArcGIS ${ }^{\circledR} 10.3$ possibilitando o armazenamento e o gerenciamento dos dados em um banco de dados criado no próprio software, facilitando se trabalhar as cenas e outros dados.

O procedimento metodológico foi baseado no proposto por Silva (2015), mas modificado para atender a demanda da localidade, o processo metodológico está sintetizado na figura 2 , sendo esmiuçados com mais detalhes nos tópicos abaixo.

\subsection{Planejamento de voo}

Nesta etapa foi definido o alvo a ser sobrevoado, a mancha urbana de Palmeirina, com uma área de $1,98 \mathrm{~km}^{2}$, representando $1,25 \%$ do município, tenho seu perímetro urbano cotas altimétricas variando entre 531 a 588.

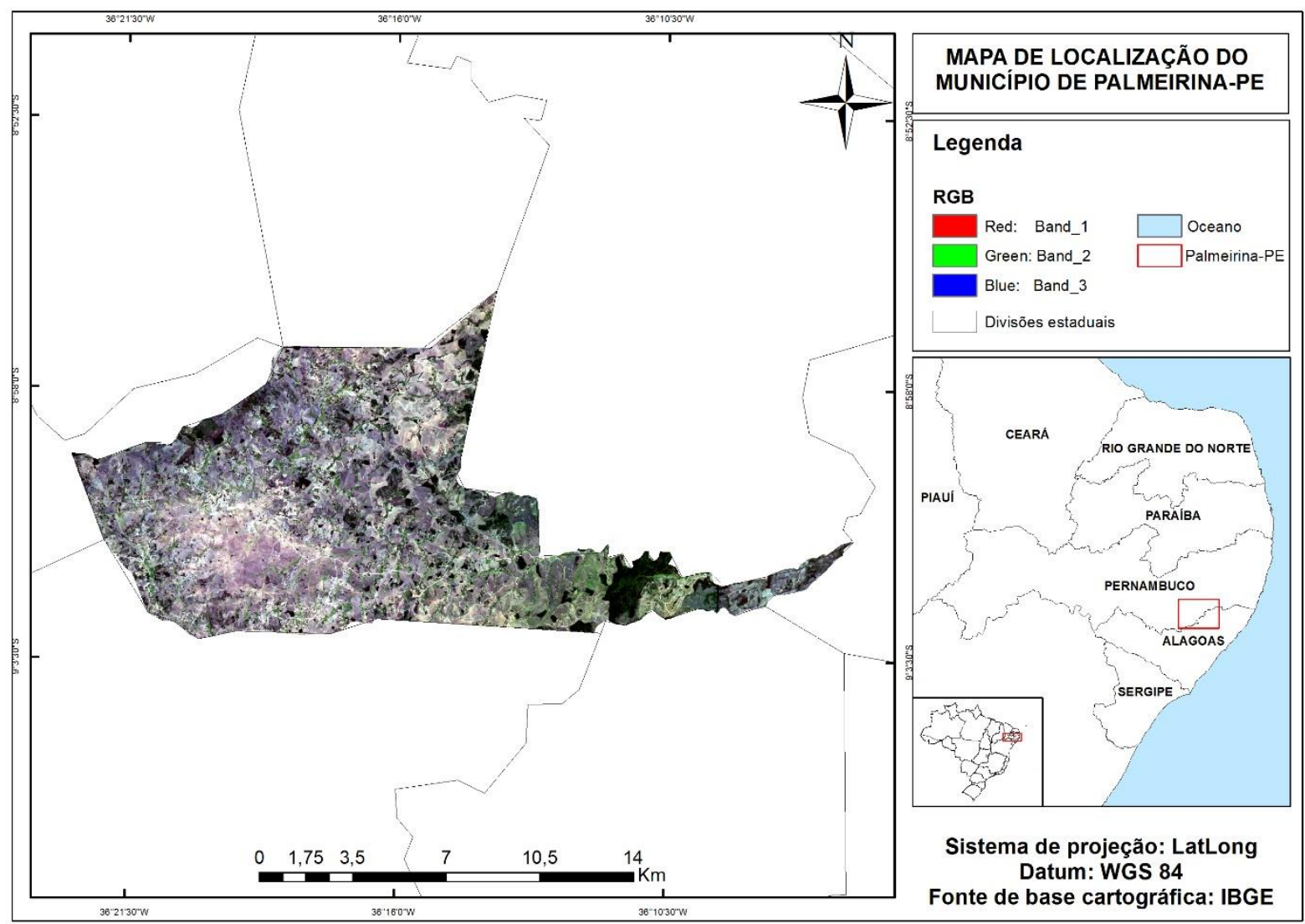

Figura 1. Mapa de localização do município de Palmeirina-PE.

Devido ao Phantom 3 Advanced ser um multimotor, não há a necessidade de catapulta para seu lançamento, já que o mesmo decola verticalmente, facilitando assim iniciar o voo em local de difícil mobilidade. A partir da decolagem em modo manual até a altitude de 50 metros, há o acionamento do piloto automático, que irá elevar a aeronave até 100 metros e iniciar o plano de voo e a tomada das 
OS DESAFIOS DA GEOGRAFIA FÍSICA NA FRONTEIRA DO CONHECIMENTO

Instituto de Geociências - Unicamp

Campinas - SP

28 de Junho à 02 de Julho de 2017

cenas, sendo que nenhum voo ultrapassou o tempo de 15 minutos, por motivos de segurança, já que a bateria dura em média 23 minutos de voo. Logo após concluído o voo, o VANT retorna até o ponto de decolagem, por meio do Go to home, pousando de modo vertical, em um raio de 3 metros do ponto de decolagem. Pode-se perceber com mais detalhes referentes a cada voo na tabela 1.

\subsection{Pós voo}

Finalizada a execução do voo, ocorre a avaliação das fotografias obtidas e da rota do GPS, avaliando o contraste e a nitidez, enquanto que na rota se verifica se o VANT não se distanciou demais da rota planejada devido ao vento. Após a verificação dos dados, as cenas foram armazenadas em um Geodatabase no ArcGIS 10.3.

Após os dados armazenados em um Banco de Dados (BD), utilizou-se o software Argisoft PhotoScan Professional, para gerar o ortomosaicos e aplicação dos pontos de controle, levando cerca de 22 horas de processamento, obtendo um ortomosaico com $4,23 \mathrm{~cm} /$ pix de resolução espacial.

As cinco etapas básicas do processameo nto com o Photoscan são: (1) calibração automática da câmera - com base nos dados do EXIF das fotografias; (2) alinhamento das fotos - a partir dos pontos em comum entre as fotografias; (3) geração da nuvem de pontos - com base nas posições estimadas das fotografias são identificadas as coordenadas x, y e z; (4) criação de um MDS de malha triangular - usando a nuvem de pontos como nós, é gerada uma estrutura do tipo vetorial com topologia do tipo nó-arco que representa a superfície através de um conjunto de faces triangulares interligadas; (5) geração do ortomosaico - gerado a partir da texturização da geometria construída pela malha triangular.

\subsection{Criação da base cartográfica}

Posteriormente a exportação do ortomosaico no formato GeoTiff (.tif), utilizou-se o ArcGIS 10.3, que possibilitou a criação de novos shapefiles $(s h p)$ polylines. A partir da criação do Shp iniciou-se o processo de mapeamento, definiu-se como ponto de partida mapear os arruamentos, vetorizando todos os meios-fios, posterior a essa etapa foi feito a cartografia de todos os imóveis da área urbana, sejam eles construídos ou em construção. Após as construções humanas, foram mapeadas as áreas verdes e recursos hídricos (rio que banha a cidade), todo a cartografia feita na escala de 1:500, devido ao grande nível de detalhes proporcionada pela aerofotogrametria (DUARTE, 2013). Os procedimentos executados foram aplicados para desenvolver metodologias e obter os resultados almejados, a figura 3 apresenta o ortomosaico gerado na etapa 2.4.

\section{Resultados e discussões}




\section{OS DESAFIOS DA GEOGRAFIA FÍSICA NA FRONTEIRA DO CONHECIMENTO \\ Instituto de Geociências - Unicamp \\ Campinas - SP \\ 28 de Junho à 02 de Julho de 2017}

Embora o VANT Phantom 3 Advanced seja um VANT de pequeno porte, o mesmo desempenhou bem a proposta de gerar um ortomosaico da mancha urbana de Palmeirina-PE. Mas só foi possível devido a área ser pequena e o grande números de voos, conhecendo as limitações e capacidades do VANT, não se recomenda o mesmo para o levantamento de grandes áreas.

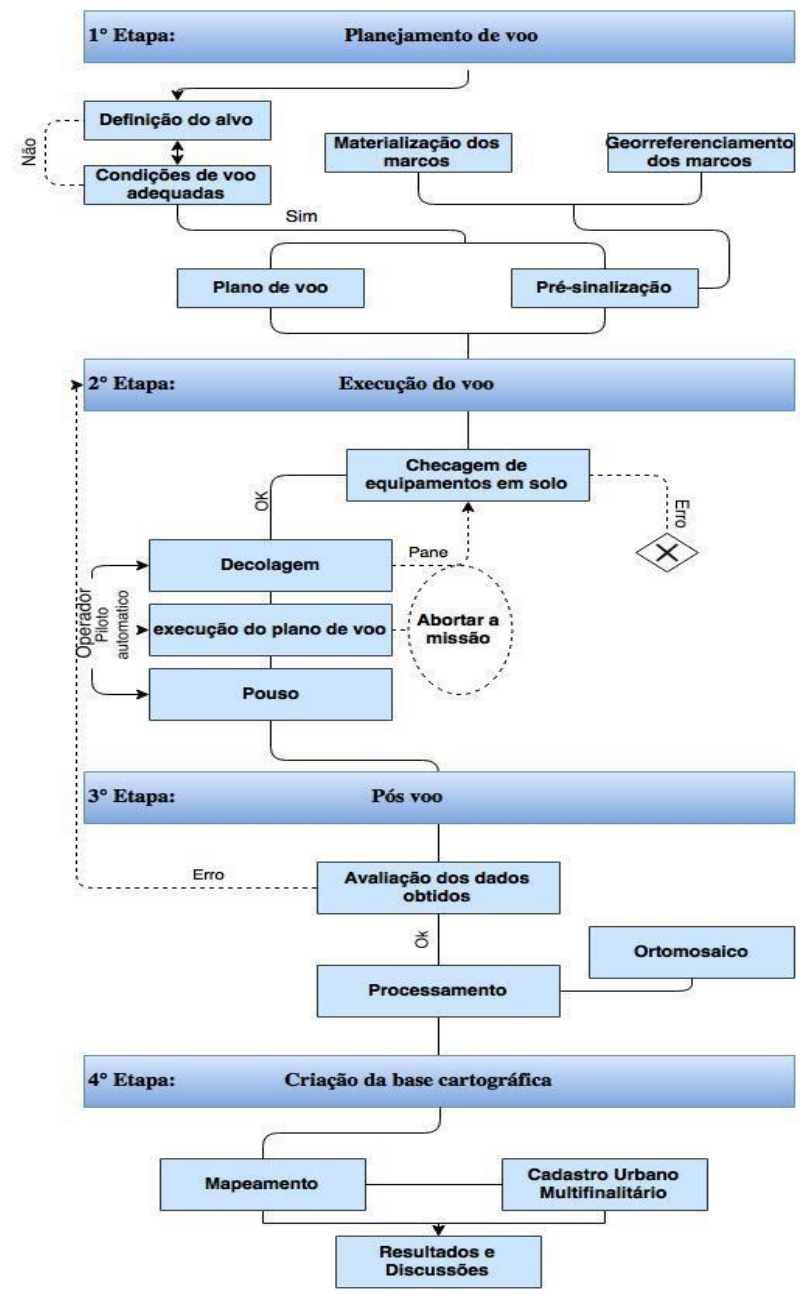

Figura 2. Fluxograma com as etapas metodológicas. 
XVII Simpósio Brasileiro de Geografia Física Aplicada

I Congresso Nacional de Geografia Física
OS DESAFIOS DA GEOGRAFIA FÍSICA NA FRONTEIRA DO CONHECIMENTO

Instituto de Geociências - Unicamp

Campinas - SP

28 de Junho à 02 de Julho de 2017

Tabela 1. Detalhes acerca de cada levantamento em campo.

\begin{tabular}{|c|cccc}
$\begin{array}{c}\text { Número Do } \\
\text { Voo }\end{array}$ & Data & $\begin{array}{c}\text { Horário De } \\
\text { Decolagem }\end{array}$ & Cenas & Altura Média (M) \\
\hline FLIGHT 01 & $03 / 06 / 2016$ & $10: 45$ & 49 & 95,10 \\
FLIGHT 02 & $03 / 06 / 2016$ & $11: 20$ & 45 & 96,85 \\
FLIGHT 03 & $03 / 06 / 2016$ & $13: 50$ & 57 & 97,59 \\
FLIGHT 04 & $04 / 06 / 2016$ & $11: 05$ & 82 & 98,27 \\
FLIGHT 05 & $04 / 06 / 2016$ & $12: 56$ & 71 & 98,51 \\
FLIGHT 06 & $08 / 06 / 2016$ & $10: 14$ & 77 & 98,63 \\
FLIGHT 07 & $12 / 06 / 2016$ & $13: 23$ & 97 & 98,55 \\
FLIGHT 08 & $13 / 06 / 2016$ & $11: 58$ & 101 & 98,40 \\
FLIGHT 09 & $17 / 06 / 2016$ & $12: 34$ & 29 & 97,38 \\
FLIGHT 10 & $18 / 06 / 2016$ & $11: 23$ & 105 & 99,91 \\
FLIGHT 11 & $\mathbf{2 0 / 0 6 / 2 0 1 6}$ & $\mathbf{1 3 : 4 7}$ & $\mathbf{7 1}$ & \\
\hline
\end{tabular}

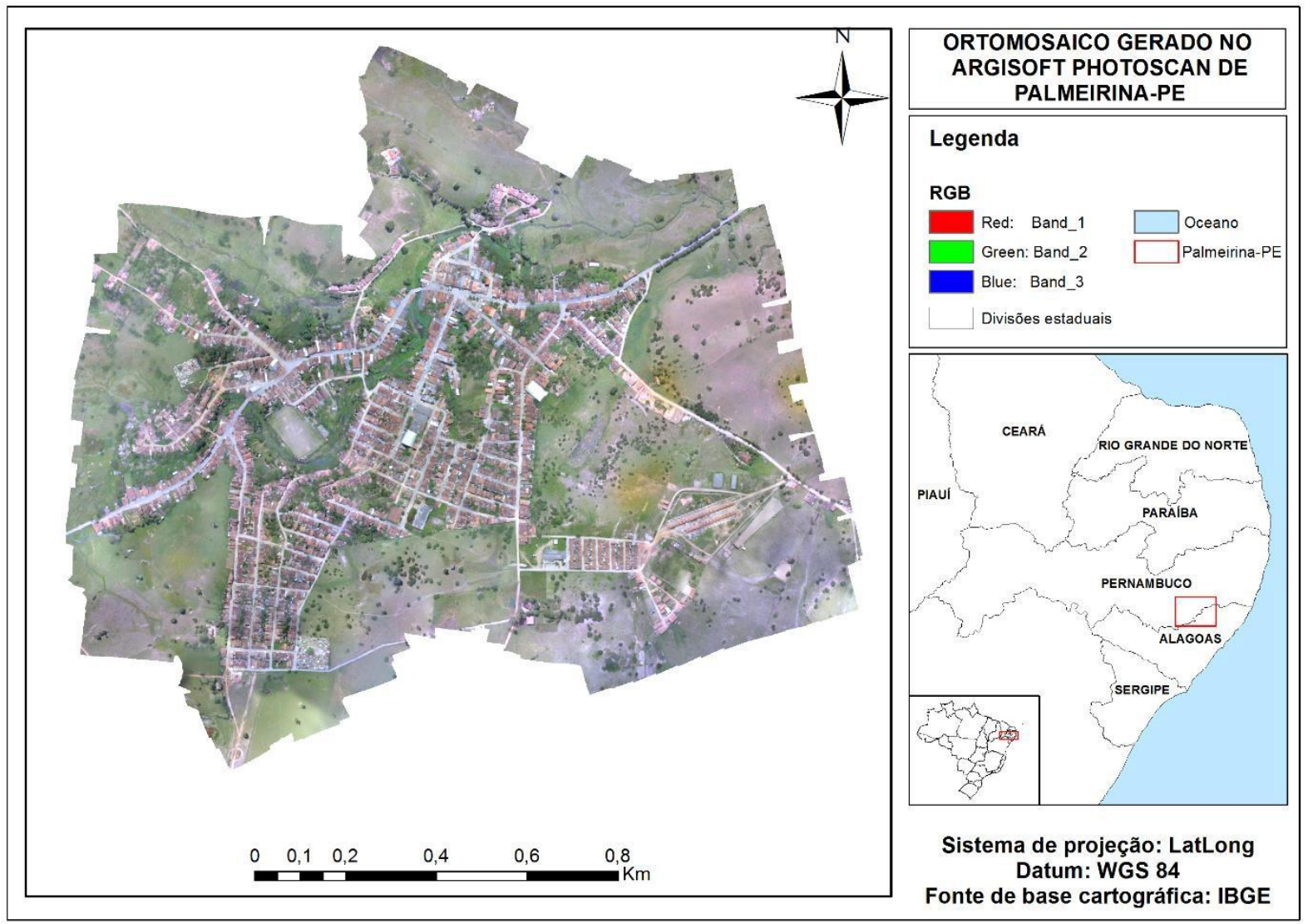

Figura 3. Ortomosaico gerado no Argisoft Photoscan de Palmeirina-PE. 


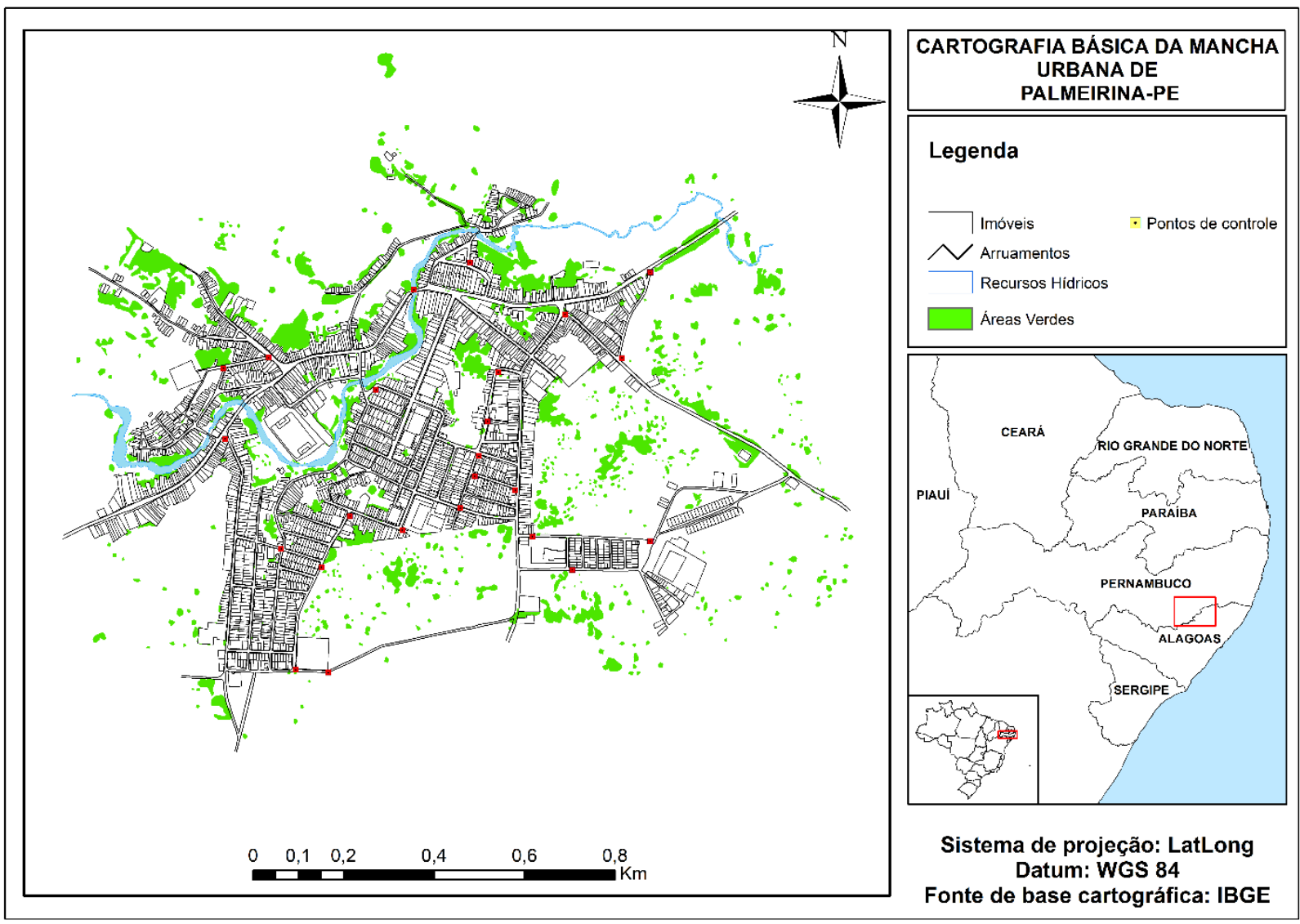

Figura 4. Cartografia básica da mancha urbana de Palmeirina-PE.

Percebeu-se um erro médio de deslocamento de 5,87 metros de raio sem os pontos de controle, que a partir da junção com os 24 pontos de controle espalhados pela mancha urbana diminuiu para 1,43 metros o raio de erro, mostrando assim a grande diferença que os pontos de controle irão fazer na realização do trabalho, mesmo sendo um GPS de navegação.

Com a correção do erro de deslocamento do ortomosaico, pode-se realizar a cartografia básica, que levou em torno de 53 horas para ser finalizada, que a partir dela os gestores públicos poderão planejar, gerir e repensar suas ações, a partir do conhecimento advindo de uma planta baixa. Que servirá não só para os tomadores de decisões, mas para toda a população, pois o conhecimento do espaço em que habitamos nos faz melhor planejadores.

A metodologia adotada possibilitou desde a definição do objeto de estudo até realização da cartografia básica, que é o primeiro passo para a consolidação de um cadastro urbano multifinalitário sólido.

Percebe-se na figura 4 o mapa final com a cartografia básica da cidade, abrangente todos os imóveis urbanos, os arruamentos, áreas verdes e os recursos hídricos. 


\section{Conclusões}

A aerofotogrametria propiciou a realização da cartografia básica da cidade de Palmeirina, com grande qualidade cartográfica, mostrando uma das grandes possibilidades de aplicação dos veículos aéreos não tripulados (VANTs). Os dados da aerofotogrametria em conjunto com o Sensoriamento Remoto é uma poderosa ferramenta para a gestão pública e dos recursos naturais.

Essa tecnologia permitiu a criação de um Sistema de Informações Geográficas (SIG) com seus arruamentos, imóveis, áreas verdes e recursos hídricos, agora em posse desses dados espera-se que os gestores públicos deem continuidade ao Cadastro Urbano Multifinalitário, para que posteriormente o planejamento e a gestão se dê de forma mais organizada.

5. Referências bibliográficas

DUARTE. P.A. Fundamentos de Cartografia. 3. Ed. Florianópolis. UFSC, 2008.

FITZ, P. R. Geoprocessamento sem complicação. 3.ed. revisada e ampliada. São Paulo. 2013. 26p.

SILVA, C. A.; DUARTE. C. R.; SOUTO. M. V. S.; SABADIA. J. A. B.: Utilização de VANT para geração de ortomosaicos e aplicação do Padrão de Exatidão Cartográfica (PEC). Anais XVII Simpósio Brasileiro de Sensoriamento Remoto - SBSR, João Pessoa-PB, Brasil, 25 a 29 de abril de 2015, INPE.

SOUZA. G.C.: Análise de metodologias no levantamento de dados espaciais para cadastro urbano. Dissertação de mestrado. Universidade de São Paulo. São Carlos. 2001 ROSA. R. Introdução ao sensoriamento remoto. 7 ed. São1994.

\section{Agradecimentos}

Os autores agradecem a Universidade de Pernambuco (UPE) pelo financiamento dos recursos do projeto de pesquisa “Análise De Riscos Socioambientais em Bacias Hidrográficas Através da Utilização de Ferramentas de Geoprocessamento e Aerofotogrametria De Pequeno Formato", junto ao Programa de Fortalecimento Acadêmico (PFA/IC), e ao Laboratório de Geoprocessamento e Modelagem Ambiental pelo apoio em todo o decorrer do trabalho. 